\title{
Comparison of Fuzzy Operators for IF-Inference Systems of Takagi-Sugeno Type in Ozone Prediction
}

\author{
Vladimír Olej and Petr Hájek \\ Institute of System Engineering and Informatics, \\ Faculty of Economics and Administration, \\ University of Pardubice, Studentská 84, \\ 53210 Pardubice, Czech Republic \\ \{vladimir.olej, petr.hajek\}@upce.cz
}

\begin{abstract}
The paper presents IF-inference systems of Takagi-Sugeno type. It is based on intuitionistic fuzzy sets (IF-sets), introduced by K.T. Atanassov, fuzzy $\mathrm{t}$-norm and t-conorm, intuitionistic fuzzy t-norm and t-conorm. Thus, an IFinference system is developed for ozone time series prediction. Finally, we compare the results of the IF-inference systems across various operators.
\end{abstract}

Keywords: Fuzzy inference systems, IF-sets, IF-inference system of TakagiSugeno type, t-norm, t-conorm, intuitionistic fuzzy t-norm and t-conorm, time series, ozone prediction.

\section{Introduction}

The IF-sets theory [1] represents one of the generalizations, the notion introduced by K.T. Atanassov [2]. The concept of IF-sets can be viewed as an alternative approach to define a fuzzy set in cases where available information is not sufficient for the definition of an imprecise concept by means of a conventional fuzzy set. In this paper IF-sets will be presented as a tool for reasoning in the presence of imperfect facts and imprecise knowledge. The IF-sets have been for example applied for ozone prediction [3] and air quality modelling as they provide a good description of object attributes by means of membership functions $\mu$ and non-membership functions $v$. They also present a strong potential to express uncertainty. In our previous paper [3] a novel IFinference system of Takagi-Sugeno type was presented for time series prediction. In this paper, the outputs of the IF-inference system are compared across various operators in if-then rules.

This paper presents basic notions of Takagi-Sugeno type fuzzy inference systems (FIS) for time series prediction. Based on the FIS defined in this way and IF-sets, $t-$ norms and t-conorms, intuitionistic fuzzy t-norms and t-conorms are defined. Next, IF-inference systems of Takagi-Sugeno type are introduced. Further, the paper includes a comparison of the prediction results obtained by the FIS characterized by membership functions $\mu$, by the FIS characterized by non-membership functions $v$, and by the IF-inference system. The comparison is realized for the example of ozone prediction in the Pardubice micro region, the Czech Republic. 


\section{IF-Inference System of Takagi-Sugeno Type}

General structure of FIS is defined in [4]. It contains a fuzzification process of input variables by membership functions $\mu$, the design of the base of if-then rules or automatic extraction of if-then rules from input data, application of operators (AND,OR,NOT) in if-then rules, implication and aggregation within these rules, and the process of defuzzification of gained values to crisp values.

In [4] there are mentioned optimization methods of the number of if-then rules. Operator AND between elements of two fuzzy sets can be generalized by t-norm and operator OR between elements of two fuzzy sets can be generalized by t-conorm [5]. The concept of IF-sets is the generalization of the concept of fuzzy sets, the notion introduced by L.A. Zadeh [5]. The theory of IF-sets is well suited to deal with vagueness. Recently, in this context, IF-sets have been used for intuitionistic classification and prediction models which can accommodate imprecise information. Based on [2], using membership function $\mu_{A}(x)$, non-membership function $v_{A}(x)$ and intuitionistic fuzzy index (IF-index) $\pi_{A}(x)$ it is possible to define an IF-system. The value $\pi_{A}(x)$ denotes a measure of non-determinancy, it may cater to either membership value or non-membership value, or both.

Let there exist a general IF-system defined in [6]. Then it is possible to define its output $y^{\eta}$ as $y^{\eta}=\left(1-\pi_{A}(x)\right) \times y^{\mu}+\pi_{A}(x) \times y^{\nu}$, where $y^{\mu}$ is the output of the FIS $^{\mu}$ using the membership function $\mu_{A}(x), y^{v}$ is the output of the FIS ${ }^{v}$ using the non-membership function $v_{A}(x)$. For the IF-inference system designed in this way, the following facts hold. If IF-index [3]:

- $\pi_{A}(x)=0$, then the output of IF-inference system $y^{\eta}=\left(1-\pi_{A}(x)\right) \times y^{\mu}$ (TakagiSugeno type FIS is characterized by membership function $\mu$ ).

- $\pi_{A}(x)=1$, then the output of IF-inference system $y^{\eta}=\pi_{A}(x) \times y^{v}$ (Takagi-Sugeno type FIS is characterized by non-membership function $v$ ).

- $0<\pi_{A}(x)<1$, then the output of IF-inference system $y^{\eta}=\left(1-\pi_{A}(x)\right) \times y^{\mu}+\pi_{A}(x)$ $\times y^{v}$ (Takagi-Sugeno type FIS is characterized by membership function $\mu$ and non-membership function $v$ ).

Let $x_{1}, x_{2}, \ldots, x_{j}, \ldots, x_{m}$ be input variables FIS ${ }^{\eta}$ defined on reference sets $X_{1}, X_{2}, \ldots, X_{j}, \ldots$ ,$X_{m}$ and let $y^{\eta}$ be an output variable defined on reference set $Y$. Then FIS ${ }^{\eta}$ has $m$ input variables $x_{1}, x_{2}, \ldots, x_{j}, \ldots, x_{m}$ and one output variable $y^{\eta}$, where $\eta=\mu$ are membership functions ( $\eta=v$ are non-membership functions).

Further, each set $X_{j}, j=1,2, \ldots, m$, can be divided into $i=1,2, \ldots, n$ fuzzy sets which are represented by following way $\eta_{j, 1}(x), \eta_{j, 2}(x), \ldots, \eta_{j, i}(x), \ldots, \eta_{j, n}(x)$. Individual fuzzy sets, where $\eta=\mu$ are membership functions $(\eta=v$ are non-membership functions) represent a mapping of linguistic variables values which are related to sets $X_{j}$. Then the $k$-th if-then rule $R_{k}$ in FIS $^{\eta}$ can be defined as follows

$$
\begin{gathered}
R_{k}: \text { if } x_{1} \text { is } A_{1, i(1, k)}{ }^{\eta} \text { AND } x_{2} \text { is } A_{2, i(2, k)}{ }^{\eta} \text { AND } \ldots \text { AND } x_{j} \text { is } A_{j, i(j, k)}{ }^{\eta} \text { AND } \ldots \text { AND } \\
x_{m} \text { is } A_{m, i(m, k)}{ }^{\eta} \text { then } y^{\eta}=h \text {, or } y^{\eta}=f\left(x_{1}, x_{2}, \ldots, x_{m}\right), j=1,2, \ldots, m ; i=1,2, \ldots, n,
\end{gathered}
$$

where $A_{1, i(1, k)}{ }^{\eta}, A_{2, i(2, k)}{ }^{\eta}, \ldots, A_{j, i(j, k)}{ }^{\eta}, \ldots, A_{m, i(m, k)}{ }^{\eta}$ represent the values of linguistic variable for $\mathrm{FIS}^{\mu}$ and $\mathrm{FIS}^{v}, h$ is constant, $f\left(x_{1}, x_{2}, \ldots, x_{m}\right)$ is a linear or polynomial function. 


\section{Intuitionistic Fuzzy t-Norms and t-Conorms}

Let there be given two fuzzy sets which are defined in the same universe. Then it is possible to realize operations between them, i.e. such forms of projections (relations) where the result projects to the same universe. Within the fuzzy sets theory it is possible to define classes of operations where intersection is corresponded by t-norms and union by t-conorms. Therefore, definitions of the most important fuzzy sets operations are listed further [4,7]. As well as union and intersection are dual operations, also t-norms and t-conorms are dual. Their relation can be defined as

$$
T(x, y)=1-S((1-x),(1-y)), S(x, y)=1-T((1-x),(1-y)) .
$$

Based on the introduced facts, it is possible to define stated t-norms and t-conorms which always create pairs (one t-norm and one t-conorm). Examples of t-norms and tconorms are listed in Table 1. The analysis in [7] implies that t-norms and t-conorms are characterized by different rate of strictness, or tolerance, where $T_{D}$ is the strictest tnorms and $S_{D}$ the most tolerant t-conorm. They can be ordered in the following way

$$
T_{D} \leq T_{L} \leq T_{E} \leq T_{A} \leq T_{H} \leq T_{M} \text { and } S_{M} \leq S_{H} \leq S_{A} \leq S_{E} \leq S_{L} \leq S_{D}
$$

Fuzzy sets have associated a non-membership function $v_{A}(x)$ degree

$$
A=\left\{\left\langle x, \mu_{A}(x), v_{A}(x)\right\rangle \mid x \in X\right\}=\left\{\left\langle x, \mu_{A}(x), 1-\mu_{A}(x)\right\rangle \mid x \in X\right\} .
$$

\begin{tabular}{|c|c|c|c|}
\hline Name & & t-norm, t-conorm & Notes \\
\hline Drastic & $\begin{array}{l}\text { product } \\
\text { sum }\end{array}$ & $\begin{aligned} T_{D}(x, y)= & \min (x, y) \text { if } \max (x, y)=1 \\
& 0 \text { otherwise } \\
S_{D}(x, y)= & \max (x, y) \text { if } \min (x, y)=0 \\
& 1 \text { otherwise }\end{aligned}$ & \\
\hline Bounded & $\begin{array}{l}\text { difference } \\
\text { sum }\end{array}$ & $\begin{array}{l}T_{L}(x, y)=\max (0, x+y-1) \\
S_{L}(x, y)=\min (1, x+y)\end{array}$ & $\begin{array}{l}\text { Łukasiewicz t-norm } \\
\text { Łukasiewicz t-conorm }\end{array}$ \\
\hline Einstein & $\begin{array}{l}\text { product } \\
\text { sum }\end{array}$ & $\begin{array}{l}T_{E}(x, y)=(x \cdot y) /((2-(x+y-x \cdot y)) \\
S_{E}(x, y)=(x+y) /(1+x \cdot y)\end{array}$ & \\
\hline $\begin{array}{l}\text { Algebraic } \\
\text { Probabilistic }\end{array}$ & $\begin{array}{l}\text { product } \\
\text { sum }\end{array}$ & $\begin{array}{l}T_{A}(x, y)=x \cdot y \\
S_{A}(x, y)=x+y-x \cdot y\end{array}$ & $\begin{array}{l}\text { Algebraic product } \\
\text { Probabilistic sum }\end{array}$ \\
\hline Hamacher & $\begin{array}{l}\text { product } \\
\text { sum }\end{array}$ & $\begin{array}{l}T_{H}(x, y)=(x \cdot y) /(x+y-x . y) \\
S_{H}(x, y)=(x+y-2 . x \cdot y) /(1-x . y)\end{array}$ & \\
\hline MIN, MAX & $\begin{array}{l}\min \\
\max \end{array}$ & $\begin{array}{l}T_{M}(x, y)=\min (x, y) \\
S_{M}(x, y)=\max (x, y)\end{array}$ & $\begin{array}{l}\text { Gödel t-norm } \\
\text { Gödel t-conorm }\end{array}$ \\
\hline
\end{tabular}

Table 1. Examples of t-norms and t-conorms

Let be given an automorphism [8] of the unit interval, i.e. every function $\psi$ : $[0,1] \rightarrow[0,1]$, that is continuous and strictly increasing such that $\psi(0)=0$ and $\psi(1)=1$. Further, let be given a function $n:[0,1] \rightarrow[0,1]$ in such a way that it holds $n(0)=1$ and $n(1)=0$. It is called a strong negation and it is always strictly decreasing, continuous and involutive. Then, as proved by [8], $n:[0,1] \rightarrow[0,1]$ is a strong negation if and only if there exists an automorphism $\psi$ of the unit interval such that $n(x)=\psi^{-1}(1-\psi(x))$. Let $L^{*}$ be a set for which 


$$
L^{*}=\{(x, y) \mid(x, y) \in[0,1] \times[0,1] \text { and } x+y \leq 1\}
$$

and the elements $0_{L^{*}}=(0,1)$ and $1_{L^{*}}=(1,0)$. Then $\forall((x, y),(z, t)) \in L^{*}$ it holds:

$-(x, y) \leq_{L^{*}}(z, t)$ iff $x \leq z$ and $y \geq t$. This relation is transitive, reflexive and antisymmetric.

$$
-(x, y)=(z, t) \text { iff }(x, y) \leq_{\mathrm{L}^{*}}(z, t) \text { and }(z, t) \leq_{L^{*}}(x, y),(x, y) \preccurlyeq(z, t) \text { iff } x \leq z \text { and } y \leq t .
$$

The designed IF-inference system of Takagi-Sugeno type works with the inference mechanism, based on intuitionistic fuzzy t-norm and t-conorm, by means of t-norm and t-conorm [9] on interval [0,1]. A function $T:\left(L^{*}\right)^{2} \rightarrow L^{*}$ is called intuitionistic fuzzy t-norm if it is commutative, associative, and increasing in both arguments with respect to the order $\leq_{L^{*}}$ and with neutral element $1_{L^{*}}$. Similarly, a function $S:\left(L^{*}\right)^{2} \rightarrow$ $L^{*}$ is called intuitionistic fuzzy t-conorm if it is commutative, associative, and increasing with neutral element $0_{L^{*}}$. Intuitionistic fuzzy t-norm $\boldsymbol{T}$ is called $\mathrm{t}-$ representable if and only if there exists a t-norm $T$ and t-conorm $S$ on interval [0,1] such that $\forall(x, y),(z, t) \in L^{*}$ it holds $\boldsymbol{T}((x, y),(z, t))=(T(x, z), S(y, t)) \in L^{*}$.

Intuitionistic fuzzy t-norm and t-conorm can be constructed using t-norms and $\mathrm{t}$ conorms on $[0,1]$ in the following way.

Let $T$ be a t-norm and $S$ a t-conorm. Then the dual t-norm $S^{*}$ of $S$ is defined by $S^{*}(x, y)=1-S(1-x, 1-y), \forall x, y \in[0,1]$. If $T \leq S^{*}$, i.e. if $\forall x, y \in[0,1], T(x, y) \leq S^{*}(x, y)$, then the mapping $\boldsymbol{T}$ defined by $\boldsymbol{T}(x, y)=\left(T\left(x_{1}, y_{1}\right), S\left(x_{2}, y_{2}\right)\right), \forall x, y \in L^{*}$, is an intuitionistic fuzzy t-norm and the mapping $\boldsymbol{S}$ defined by $\boldsymbol{S}(x, y)=\left(S\left(x_{1}, y_{1}\right), T\left(x_{2}, y_{2}\right)\right), \forall x, y \in L^{*}$, is an intuitionistic fuzzy t-conorm. Note that condition $T \leq S^{*}$ is necessary and sufficient for $\boldsymbol{T}(x, y)$ and $\boldsymbol{S}(x, y)$ to be elements of $L^{*} \forall x, y \in L^{*}$. It can be written that $\boldsymbol{T}=(T, S)$ and $S=(S, T)$.

\section{Modelling and Analysis of the Results}

Verification of behaviour of various t-norms and t-conorms in designed IF-inference system of Takagi-Sugeno type is shown in ozone modelling. The data for our investigations was obtained from the Czech Hydro-meteorological Institute. This data contains the average daily ozone measurements and the average daily meteorological variables (such as temperature, wind speed, wind direction, humidity, air pressure and solar radiation), vehicle emission variables $\left(\mathrm{NO}_{2}, \mathrm{CO}, \mathrm{NO}, \mathrm{NO}_{x}, \mathrm{SO}_{2}, \mathrm{PM}_{10}\right.$ and $\mathrm{PM}_{2.5}$ ), and other dummy variables (working day, month). All the measured variables used in this study are actual same day values. Then the formulation of the basic model is following: $y=f\left(x_{1}{ }^{t}, x_{2}{ }^{t}, \ldots, x_{m}{ }^{t}\right), m=22$, where $y$ is daily average ozone level at time $t+1$. For further modelling it is necessary to minimize the number of parameters $x_{m}{ }^{t}$, $m=22$, and to maximize function $f$.

Based on the minimization of the number of parameters $x_{m}{ }^{t}, m=22$ using Pearson's correlation coefficient, it was shown that $\mathrm{NO}_{x}, \mathrm{NO}, \mathrm{NO}_{2}$, month of measurement, humidity, solar radiation, and the ozone level at day ahead were important to predict daily average ozone levels. The general formulation of the model is as follows $y=$ $f\left(x_{1}{ }^{t}, x_{2}{ }^{t}, \ldots, x_{m}{ }^{t}\right), m=7$, where $y$ is daily average ozone level at time $t+1, x_{1}{ }^{t}$ is oxide of nitrogen, $x_{2}{ }^{t}$ is nitric oxide, $x_{3}{ }^{t}$ is nitrogen dioxide, $x_{4}{ }^{t}$ is dummy variable (month), $x_{5}{ }^{t}$ is humidity, $x_{6}{ }^{t}$ is solar radiation, $x_{7}{ }^{t}$ is daily average ozone level at time $t$. 
Based on the given facts, such $\mathrm{FIS}^{\mu}$ is designed which is characterized by means of membership function $\mu$, and FIS ${ }^{v}$ characterized by non-membership function $v$. Input variables $x_{1}{ }^{t}, x_{2}{ }^{t}, \ldots, x_{m}{ }^{t}, m=7$ in time $t$ are represented by two bell-shaped membership functions $\mu$ for $\mathrm{FIS}^{\mu}$ and two non-membership functions $v$ for $\mathrm{FIS}^{v}$. Membership function $\mu$ and non-membership function $v$, and if-then rules were designed using subtractive clustering algorithm. After projecting the clusters onto the input space, the antecedent parts of the fuzzy if-then rules can be found. The consequent parts of the if-then rules are represented by polynomial functions $y=f\left(x_{1}, x_{2}, \ldots, x_{r}\right)$. In this way, one cluster corresponds to one if-then rule. To be specific, two if-then rules are designed for FIS $^{\mu}$ and FIS ${ }^{\nu}$ respectively. The output level $y_{k}$ of each of the $k$-th if-then rule $R_{k}$ is weighted. The final outputs $y^{\mu}$ and $y^{v}$ of the FIS ${ }^{\mu}$ and FIS ${ }^{v}$ are the weighted averages of all the if-then rule $R_{k}$ outputs $y_{k}, k=1,2, \ldots, N$. The output of IF-inference system is represented by the predicted value $y^{\eta}$ in time $t+1$. Table 2 shows the quality of ozone prediction represented by Root Mean Squared Error $\left(\mathrm{RMSE}^{\eta}\right)$ for different values of $\mu_{\max }$ [3], different values of IF-index $\pi$ and for various t-norms. The resulting errors are comparable to those presented in other studies, for example [10].

Table 2. $\mathrm{RMSE}^{\eta}$ on testing data $O_{\text {test }}$ for different values of $\mu_{\max }$, IF-index $\pi$, and $T_{E}, T_{A}, T_{H}, T_{M}$

\begin{tabular}{|c|c|c|c|c|c|c|c|c|c|}
\hline$\pi=0.1$ & & & & & & & & & \\
\hline$\mu_{\max }$ & 0.1 & 0.2 & 0.3 & 0.4 & 0.5 & 0.6 & 0.7 & 0.8 & 0.9 \\
\hline$T_{E}$ & 23.23 & 24.00 & 25.30 & 26.96 & 28.64 & 30.78 & 34.60 & 42.24 & 21.43 \\
\hline$T_{A}$ & 23.08 & 23.55 & 24.17 & 25.01 & 26.19 & 27.85 & 30.46 & 35.07 & 19.81 \\
\hline$T_{H}$ & 16.99 & 18.02 & 18.85 & 19.33 & 19.35 & 18.83 & 17.74 & 15.95 & 13.31 \\
\hline$T_{M}$ & - & 11.01 & 11.06 & 11.06 & 11.06 & 11.06 & 11.05 & 11.04 & 10.94 \\
\hline \multicolumn{10}{|l|}{$\pi=0.2$} \\
\hline$T_{E}$ & 22.09 & 24.05 & 27.27 & 31.29 & 36.04 & 43.92 & 60.82 & 30.27 & \\
\hline$T_{A}$ & 21.65 & 22.70 & 24.30 & 26.69 & 30.25 & 35.78 & 46.63 & 25.14 & \\
\hline$T_{H}$ & 16.33 & 17.37 & 18.30 & 18.98 & 19.21 & 18.78 & 17.12 & 13.41 & \\
\hline$T_{M}$ & - & 10.90 & 10.93 & 10.93 & 10.92 & 10.92 & 10.91 & 11.63 & \\
\hline & & & & & & & & & \\
\hline \multicolumn{10}{|l|}{$\pi=0.8$} \\
\hline$T_{E}$ & 42.26 & 112.1 & & & & & & & \\
\hline$T_{A}$ & 37.99 & 108.1 & & & & & & & \\
\hline$T_{H}$ & 15.64 & 25.08 & & & & & & & \\
\hline$T_{M}$ & 12.22 & 26.78 & & & & & & & \\
\hline
\end{tabular}

Extreme situations of the IF-inference system are to be found when IF-index $\pi_{A}(x)$ $=0$ (Table 3 ), then $\mu_{A}(x)+v_{A}(x)=1$ (fuzzy sets are considered as a particular case IFsets) and if IF-index $\pi_{A}(x)=1$, then $\mu_{A}(x)=0$ and $v_{A}(x)=0$ (complete ignorance of the problem). For $\pi_{A}(x)=0, \mathrm{RMSE}^{\mu}=\mathrm{RMSE}^{\eta}$. Thus, only $\mathrm{RMSE}^{\mu}$ are reported. Drastic $T_{D}$ and bounded $T_{L}$ t-norms are not suitable for this IF-inference system design. The problem with drastic $T_{D}$ and bounded $T_{L}$ t-norms is that it can happen that no rule is activated. When no rule is activated, the output of the system is constant, which can be interpreted as some kind of neutral output. Strictness of these t-norms is crucial for this type of data. Based on designed IF-inference systems with t-norms $T_{D}$ and $T_{L}$, it is implied that $\mathrm{RMSE}^{\eta}$ for $\mathrm{FIS}^{\eta}$ is constant. For other designed IF-inference systems with t-norms $T_{E}, T_{A}, T_{H}, T_{M}, \mathrm{RMSE}^{\eta}$ for $\mathrm{FIS}^{\eta}$ are given by Table 2 . 
Table 3. RMSE on testing data $O_{\text {test }}$ for different values of $\mu_{\max }$, IF-index $\pi=0$, and $T_{E}, T_{A}, T_{H}, T_{M}$

\begin{tabular}{|c|c|c|c|c|c|c|c|c|c|}
\hline$\mu_{\max }$ & 0.1 & 0.2 & 0.3 & 0.4 & 0.5 & 0.6 & 0.7 & 0.8 & 0.9 \\
\hline$T_{E} \mathrm{RMSE}^{\mu}$ & 24.72 & 24.69 & 24.65 & 24.57 & 24.47 & 24.34 & 24.19 & 24.03 & 23.92 \\
\hline $\mathrm{RMSE}^{v}$ & 15.94 & 15.07 & 30.33 & 56.82 & 81.50 & 106.00 & 139.60 & 196.30 & 292.60 \\
\hline$T_{A} \mathrm{RMSE}^{\mu}$ & 24.72 & 24.72 & 24.72 & 24.72 & 24.72 & 24.72 & 24.72 & 24.72 & 24.71 \\
\hline $\mathrm{RMSE}^{v}$ & 16.65 & 16.02 & 19.93 & 29.51 & 42.78 & 59.53 & 81.41 & 115.70 & 169.90 \\
\hline$T_{H} \mathrm{RMSE}^{\mu}$ & 17.70 & 18.59 & 19.20 & 19.44 & 19.22 & 18.54 & 17.42 & 16.05 & 14.76 \\
\hline RMSE $^{v}$ & 15.76 & 16.75 & 20.53 & 24.35 & 27.18 & 28.84 & 29.08 & 27.06 & 19.59 \\
\hline$T_{M} \mathrm{RMSE}^{\mu}$ & - & 11.29 & 11.29 & 11.29 & 11.29 & 11.29 & 11.29 & 11.29 & 11.29 \\
\hline $\mathrm{RMSE}^{\nu}$ & - & 13.92 & 13.25 & 13.36 & 13.33 & 13.30 & 13.31 & 13.40 & 13.70 \\
\hline
\end{tabular}

\section{Conclusion}

The development of effective prediction models of ozone concentrations in urban areas is important. However, it is difficult because the meteorological parameters and photochemical reactions involved in ozone formation are complex. The article compares IF-inference systems of Takagi-Sugeno type [3] for various fuzzy operators in if-then rules with the help of $\mathrm{RMSE}^{\eta}$ for $\mathrm{FIS}^{\eta}$ on testing data $O_{\text {test }}$. RMSE represents the error of IF-inference system, which consists of $\mathrm{RMSE}^{\mu}$ for $\mathrm{FIS}^{\mu}$, which is represented by membership function $\mu$, and error $\operatorname{RMSE}^{v}$ for FIS ${ }^{v}$, which is represented by non-membership function $v$. Further, extreme situations in the designed IF-inference systems from the viewpoint of IF-index are shown.

Acknowledgments. This work was supported by the scientific research project of the Ministry of Environment, the Czech Republic under Grant No: SP/4i2/60/07.

\section{References}

[1] Dubois, D., Gottwald, S., Hajek, P., Kacprzyk, J., Prade, H.: Terminological Difficulties in Fuzzy Set Theory-The case of Intuitionistic Fuzzy Sets. Fuzzy Sets and Systems 156, 485-491 (2005)

[2] Atanassov, K.T.: Intuitionistic Fuzzy Sets. Fuzzy Sets and Systems 20, 87-96 (1986)

[3] Olej, V., Hájek, P.: IF-Inference Systems Design for Prediction of Ozone Time Series: The Case of Pardubice Micro-Region. In: Diamantaras, K., Duch, W., Iliadis, L.S. (eds.), pp. 1-11. Springer, Heidelberg (2010)

[4] Pedrycz, W.: Fuzzy Control and Fuzzy Systems. John Wiley and Sons, New York (1993)

[5] Zadeh, L.A.: Fuzzy Sets. Inform. and Control 8, 338-353 (1965)

[6] Montiel, O., et al.: Mediative Fuzzy Logic: A new Approach for Contradictory Knowledge Management. Soft Computing 20, 251-256 (2008)

[7] Klement, E.P., Mesiar, R., Pap, E.: Triangular Norms. Position Paper I: basic Analytical and Algebraic Properties. Fuzzy Sets and Systems 143, 5-26 (2004)

[8] Barrenechea, E.: Generalized Atanassov's Intuitionistic Fuzzy Index. Construction Method. In: IFSA-EUSFLAT, Lisbon, pp. 478-482 (2009)

[9] Deschrijver, G., Cornelis, C., Kerre, E.: On the Representation of Intuitionistic Fuzzy tnorm and t-conorm. IEEE Transactions on Fuzzy Systems 12, 45-61 (2004)

[10] Comrie, A.C.: Comparing Neural Networks and Regression Models for Ozone Forecasting. Journal of the Air and Waste Management Association 47, 653-663 (1997) 